Mathematical Modelling and Analysis

Volume 17 Number 5, November 2012, 686-695

http://dx.doi.org/10.3846/13926292.2012.736088

(c) Vilnius Gediminas Technical University, 2012
Publisher: Taylor\&Francis and VGTU

http://www.tandfonline.com/TMMA

Print ISSN: 1392-6292

Online ISSN: 1648-3510

\title{
Precompactness of the Set of Trajectories of the Controllable System Described by a Nonlinear Volterra Integral Equation
}

\author{
Anar Huseyin and Nesir Huseyin \\ Anadolu University \\ 26470 Eskisehir, Turkey \\ E-mail(corresp.): ahuseyin@anadolu.edu.tr \\ E-mail: nhuseyin@anadolu.edu.tr
}

Received February 7, 2012; revised August 23, 2012; published online November 1, 2012

\begin{abstract}
In this paper the controllable system whose behaviour is described by a nonlinear Volterra integral equation, is studied. The set of admissible control functions is the closed ball of the space $L_{p}(p>1)$ with radius $\mu_{0}$ and centered at the origin. It is shown that the set of trajectories of the system is a bounded and precompact subset of the space of continuous functions.
\end{abstract}

Keywords: nonlinear Volterra integral equation, controllable system, integral constraint, set of trajectories.

AMS Subject Classification: 93C23; 45D05.

\section{Introduction}

Nonlinear integral equations arise in many problems of theory and applications (see, e.g. $[1,3,6,8,9,12]$ ). It is known that many problems of nonlinear mechanics lead to nonlinear integral equation (see, e.g. $[6,12]$ ). The process which is described by an integral equation can be under the influence of the exterior forces. If these forces are under control, then system turns out to be a controllable one. In general, control efforts are limited and therefore they have various type of constraints. Control systems with integral constraint on the controls arise in various fields of the control systems theory such as control problems with bounded $L_{p}$ norms on the controls, control problems with prescribed bounded total energy and finance, and control problems with design uncertainties (see, e.g. [4, 7, 10, 11]). For example, the motion of flying objects with variable mass, is described in the form of controllable system, where the control function has an integral constraint (see, e.g. [7, 11]). Control system with integral constraint on the controls whose behaviour is described by a nonlinear differential equation is studied in $[4,5]$. In these papers, the various topological properties and numerical construction methods of the set of 
trajectories and attainable sets of the control system with integral constraint on the controls are studied where the behaviour of the system is described by a nonlinear differential equation. In the present paper the control system with integral constraint on the controls whose behaviour is described by a nonlinear Volterra integral equation is considered. It is assumed that integral equation is nonlinear with respect to the state and control vectors. The closed ball of the space $L_{p}(p>1)$ with radius $\mu_{0}$ and centered at the origin, is chosen as the set of admissible control functions. The set of trajectories of the system generated by all admissible control functions is studied. The paper is organized as follows:

In Section 2 the basic conditions are formulated which satisfy the system (conditions 2.A, 2.B and 2.C). In Section 3 it is proved that under assumed conditions every admissible control function generates unique trajectory of the system (Theorem 1). In Section 4 the boundedness of the set of trajectories is shown (Theorem 2). In Section 5 it is proved that the sections of the set of trajectories depend on $t$ continuously (Corollary 2) and the set of trajectories is a precompact subset of the space of continuous functions (Theorem 3). It is illustrated that the set of trajectories is not a closed subset of the space of the continuous functions (Example 1).

\section{Preliminaries}

Consider the controllable system the behaviour of which is described by a nonlinear Volterra type integral equation

$$
x(t)=a(t, x(t))+\lambda \int_{t_{0}}^{t} K(t, s, x(s), u(s)) d s,
$$

where $x \in \mathbb{R}^{n}$ is the state vector of the system, $u \in \mathbb{R}^{m}$ is the control vector, $t \in\left[t_{0}, \theta\right], \lambda>0$ is a real number.

For given $p>1$ and $\mu_{0}>0$ we set

$$
U_{p}=\left\{u(\cdot) \in L_{p}\left(\left[t_{0}, \theta\right] ; \mathbb{R}^{m}\right):\|u(\cdot)\|_{p} \leq \mu_{0}\right\}
$$

where $\|u(\cdot)\|_{p}=\left(\int_{t_{0}}^{\theta}\|u(t)\|^{p} d t\right)^{\frac{1}{p}}$. The set $U_{p} \subset L_{p}\left(\left[t_{0}, \theta\right], \mathbb{R}^{m}\right)$ is called the set of admissible control functions and every function $u(\cdot) \in U_{p}$ is called admissible control function.

It is assumed that the functions $a(\cdot):\left[t_{0}, \theta\right] \times \mathbb{R}^{n} \rightarrow \mathbb{R}^{n}, K(\cdot):\left[t_{0}, \theta\right] \times$ $\left[t_{0}, \theta\right] \times \mathbb{R}^{n} \times \mathbb{R}^{m} \rightarrow \mathbb{R}^{n}$ and number $\lambda \in(0, \infty)$ given in equation (2.1) satisfy the following conditions:

2.A. The functions $a(\cdot):\left[t_{0}, \theta\right] \times \mathbb{R}^{n} \rightarrow \mathbb{R}^{n}$ and $K(\cdot):\left[t_{0}, \theta\right] \times \mathbb{R}^{n} \times \mathbb{R}^{m} \rightarrow \mathbb{R}^{n}$ are continuous;

2.B. There exist $L_{0} \in[0,1), L_{1} \geq 0, H_{1} \geq 0, L_{2} \geq 0, H_{2} \geq 0, L_{3} \geq 0$ and 
$H_{3} \geq 0$ such that

$$
\begin{aligned}
& \left\|a\left(t, x_{1}\right)-a\left(t, x_{2}\right)\right\| \leq L_{0}\left\|x_{1}-x_{2}\right\|, \\
& \quad\left\|K\left(t_{1}, s, x_{1}, u_{1}\right)-K\left(t_{2}, s, x_{2}, u_{2}\right)\right\| \leq\left[L_{1}+H_{1}\left(\left\|u_{1}\right\|+\left\|u_{2}\right\|\right)\right]\left|t_{1}-t_{2}\right| \\
& \quad+\left[L_{2}+H_{2}\left(\left\|u_{1}\right\|+\left\|u_{2}\right\|\right)\right]\left\|x_{1}-x_{2}\right\|+\left[L_{3}+H_{3}\left(\left\|x_{1}\right\|+\left\|x_{2}\right\|\right)\right]\left\|u_{1}-u_{2}\right\|
\end{aligned}
$$

for every $\left(t_{1}, s, x_{1}, u_{1}\right) \in\left[t_{0}, \theta\right] \times\left[t_{0}, \theta\right] \times \mathbb{R}^{n} \times \mathbb{R}^{m},\left(t_{2}, s, x_{2}, u_{2}\right) \in\left[t_{0}, \theta\right] \times$ $\left[t_{0}, \theta\right] \times \mathbb{R}^{n} \times \mathbb{R}^{m}$

2.C. $0 \leq \lambda\left(L_{2}\left(\theta-t_{0}\right)+2 H_{2}\left(\theta-t_{0}\right)^{\frac{p-1}{p}} \mu_{0}\right)<1-L_{0}$.

If $K(t, s, x, u)=\varphi(t, s, x)+B(t, s, x) u$ where the functions $(t, x, s) \rightarrow$ $\varphi(t, s, x)$ and $(t, s, x) \rightarrow B(t, s, x)$ are continuous with respect to $(t, s, x)$ and Lipschitz continuous with respect to $(t, x)$, then the function $K(\cdot):\left[t_{0}, \theta\right] \times$ $\left[t_{0}, \theta\right] \times \mathbb{R}^{n} \times \mathbb{R}^{m} \rightarrow \mathbb{R}^{n}$ satisfies the conditions 2.A and 2.B.

Now let us define the trajectory of the system (2.1) generated by an admissible control function $u(\cdot) \in U_{p}$.

Let $u_{*}(\cdot) \in U_{p}$. A continuous function $x_{*}(\cdot):\left[t_{0}, \theta\right] \rightarrow \mathbb{R}^{n}$ satisfying the equation

$$
x_{*}(t)=a\left(t, x_{*}(t)\right)+\lambda \int_{t_{0}}^{t} K\left(t, s, x_{*}(s), u_{*}(s)\right) d s, \quad t \in\left[t_{0}, \theta\right]
$$

is said to be a trajectory of the system (2.1) generated by the admissible control function $u_{*}(\cdot) \in U_{p}$.

We denote by $\mathbf{X}_{p}$ the set of all trajectories of the system (2.1) generated by all admissible control functions $u(\cdot) \in U_{p}$. The set $\mathbf{X}_{p}$ is called the set of trajectories of the system (2.1).

It is obvious that $\mathbf{X}_{p} \subset C\left(\left[t_{0}, \theta\right] ; \mathbb{R}^{n}\right)$ where $C\left(\left[t_{0}, \theta\right] ; \mathbb{R}^{n}\right)$ is the space of continuous functions $x(\cdot):\left[t_{0}, \theta\right] \rightarrow \mathbb{R}^{n}$ with the norm

$$
\|x(\cdot)\|_{C}=\max \left\{\|x(t)\|: t \in\left[t_{0}, \theta\right]\right\} .
$$

For each fixed $t \in\left[t_{0}, \theta\right]$ we set

$$
\mathbf{X}_{p}(t)=\left\{x(t): x(\cdot) \in \mathbf{X}_{p}\right\}
$$

According to the condition 2.B

$$
\left\|a\left(t, x_{1}\right)-a\left(t, x_{2}\right)\right\| \leq L_{0}\left\|x_{1}-x_{2}\right\|
$$

for every $t \in\left[t_{0}, \theta\right]$ and $x_{1} \in \mathbb{R}^{n}, x_{2} \in \mathbb{R}^{n}$ where $L_{0} \in[0,1)$. Then for each fixed $t \in\left[t_{0}, \theta\right]$ the function $a(t, \cdot): \mathbb{R}^{n} \rightarrow \mathbb{R}^{n}$ has a unique fixed point, i.e. for each fixed $t \in\left[t_{0}, \theta\right]$ there exists a unique $s_{t} \in \mathbb{R}^{n}$ such that

$$
s_{t}=a\left(t, s_{t}\right) .
$$

Then (2.1) and (2.4) imply that $x\left(t_{0}\right)=a\left(t_{0}, x\left(t_{0}\right)\right)=s_{t_{0}}$ for every $x(\cdot) \in \mathbf{X}_{p}$. So we obtain validity of the following proposition.

Proposition 1. The equality $\mathbf{X}_{p}\left(t_{0}\right)=\left\{s_{t_{0}}\right\}$ holds where $s_{t_{0}} \in \mathbb{R}^{n}$ is defined by $(2.4)$. 


\section{Existence and Uniqueness of the Trajectories}

Denote

$$
L(\lambda)=L_{0}+\lambda\left[L_{2}\left(\theta-t_{0}\right)+2 H_{2}\left(\theta-t_{0}\right)^{\frac{p-1}{p}} \mu_{0}\right] .
$$

The following theorem characterizes the existence and uniqueness of the trajectories of the system (2.1) generated by a given admissible control function.

Theorem 1. Let the functions $a(\cdot):\left[t_{0}, \theta\right] \times \mathbb{R}^{n} \rightarrow \mathbb{R}^{n}, K(\cdot):\left[t_{0}, \theta\right] \times\left[t_{0}, \theta\right] \times$ $\mathbb{R}^{n} \times \mathbb{R}^{m} \rightarrow \mathbb{R}^{n}$ and the number $\lambda \in(0, \infty)$ satisfy the conditions $2 . \mathrm{A}, 2 . \mathrm{B}$ and 2.C. Then for each $u_{*}(\cdot) \in U_{p}$ the system (2.1) has a unique trajectory $x_{*}(\cdot)$.

Proof. For $x(\cdot) \in C\left(\left[t_{0}, \theta\right] ; \mathbb{R}^{n}\right)$ we set

$$
F(x(\cdot)) \mid(t)=a(t, x(t))+\lambda \int_{t_{0}}^{t} K\left(t, s, x(s), u_{*}(s)\right) d s, \quad t \in\left[t_{0}, \theta\right] .
$$

Since $u_{*}(\cdot) \in U_{p}, x(\cdot) \in C\left(\left[t_{0}, \theta\right] ; \mathbb{R}^{n}\right)$ then by virtue of condition 2 .A the function $t \rightarrow F(x(\cdot)) \mid(t), t \in\left[t_{0}, \theta\right]$, is continuous and hence $F(\cdot): C\left(\left[t_{0}, \theta\right] ; \mathbb{R}^{n}\right) \rightarrow$ $C\left(\left[t_{0}, \theta\right] ; \mathbb{R}^{n}\right)$.

Let us choose arbitrary $x_{1}(\cdot) \in C\left(\left[t_{0}, \theta\right] ; \mathbb{R}^{n}\right)$ and $x_{2}(\cdot) \in C\left(\left[t_{0}, \theta\right] ; \mathbb{R}^{n}\right)$. Then applying condition 2.B we obtain

$$
\begin{aligned}
& \left\|F\left(x_{2}(\cdot)\right)\left|(t)-F\left(x_{1}(\cdot)\right)\right|(t)\right\| \leq\left\|a\left(t, x_{2}(t)\right)-a\left(t, x_{1}(t)\right)\right\| \\
& \quad+\lambda \int_{t_{0}}^{t}\left\|K\left(t, s, x_{2}(s), u_{*}(s)\right)-K\left(t, s, x_{1}(s), u_{*}(s)\right)\right\| d s \\
& \quad \leq L_{0}\left\|x_{2}(t)-x_{1}(t)\right\|+\lambda \int_{t_{0}}^{t}\left(L_{2}+2 H_{2}\left\|u_{*}(s)\right\|\right)\left\|x_{2}(s)-x_{1}(s)\right\| d s \\
& \quad \leq L_{0}\left\|x_{2}(\cdot)-x_{1}(\cdot)\right\|_{C}+\lambda \int_{t_{0}}^{t}\left(L_{2}+2 H_{2}\left\|u_{*}(s)\right\|\right)\left\|x_{2}(\cdot)-x_{1}(\cdot)\right\|_{C} d s \\
& \quad=\left(L_{0}+\lambda L_{2}\left(\theta-t_{0}\right)+2 \lambda H_{2} \int_{t_{0}}^{t}\left\|u_{*}(s)\right\| d s\right)\left\|x_{2}(\cdot)-x_{1}(\cdot)\right\|_{C}
\end{aligned}
$$

for every $t \in\left[t_{0}, \theta\right]$ where

$$
\left\|x_{2}(\cdot)-x_{1}(\cdot)\right\|_{C}=\max \left\{\left\|x_{2}(t)-x_{1}(t)\right\|: t \in\left[t_{0}, \theta\right]\right\} .
$$

Since $u_{*}(\cdot) \in U_{p}$ then using Hölder's inequality we have

$$
\int_{t_{0}}^{t}\left\|u_{*}(s)\right\| d s \leq\left(t-t_{0}\right)^{\frac{p-1}{p}}\left(\int_{t_{0}}^{t}\left\|u_{*}(s)\right\|^{p} d s\right)^{\frac{1}{p}} \leq\left(\theta-t_{0}\right)^{\frac{p-1}{p}} \mu_{0} .
$$

From (3.1), (3.3) and (3.4) it follows

$$
\begin{aligned}
& \left\|F\left(x_{2}(\cdot)\right)\left|(t)-F\left(x_{1}(\cdot)\right)\right|(t)\right\| \\
& \quad \leq\left(L_{0}+\lambda L_{2}\left(\theta-t_{0}\right)+2 \lambda H_{2}\left(\theta-t_{0}\right)^{\frac{p-1}{p}} \mu_{0}\right)\left\|x_{2}(\cdot)-x_{1}(\cdot)\right\|_{C} \\
& \quad=L(\lambda)\left\|x_{2}(\cdot)-x_{1}(\cdot)\right\|_{C}
\end{aligned}
$$


and hence

$$
\left\|F\left(x_{2}(\cdot)\right)\left|(\cdot)-F\left(x_{1}(\cdot)\right)\right|(\cdot)\right\|_{C} \leq L(\lambda)\left\|x_{2}(\cdot)-x_{1}(\cdot)\right\|_{C},
$$

where $L(\lambda)$ is defined by (3.1).

From condition 2.C we have $L(\lambda)<1$. Then by virtue of $(3.5) F(\cdot)$ : $C\left(\left[t_{0}, \theta\right] ; \mathbb{R}^{n}\right) \rightarrow C\left(\left[t_{0}, \theta\right] ; \mathbb{R}^{n}\right)$ defined by $(3.2)$ is a contractive map and consequently it has a unique fixed point $x_{*}(\cdot) \in C\left(\left[t_{0}, \theta\right] ; \mathbb{R}^{n}\right)$. Thus

$$
x_{*}(t)=a\left(t, x_{*}(t)\right)+\lambda \int_{t_{0}}^{t} K\left(t, s, x_{*}(s), u_{*}(s)\right) d s, \quad t \in\left[t_{0}, \theta\right]
$$

and the function $x_{*}(\cdot):\left[t_{0}, \theta\right] \rightarrow \mathbb{R}^{n}$ is a unique trajectory of the system $(2.1)$ generated by the admissible control function $u_{*}(\cdot) \in U_{p}$.

\section{Boundedness of the Set of Trajectories}

Let us give an auxiliary proposition which will be used in following arguments. We set

$$
\begin{aligned}
& \gamma_{0}=\max \left\{\|a(t, 0)\|: t \in\left[t_{0}, \theta\right]\right\} \\
& \gamma_{1}=\max \left\{\left\|K_{1}(t, s, 0,0)\right\|:(t, s) \in\left[t_{0}, \theta\right] \times\left[t_{0}, \theta\right]\right\} .
\end{aligned}
$$

Proposition 2. Let the functions $a(\cdot, \cdot):\left[t_{0}, \theta\right] \times \mathbb{R}^{n} \rightarrow \mathbb{R}^{n}$ and $K(\cdot, \cdot, \cdot, \cdot)$ : $\left[t_{0}, \theta\right] \times\left[t_{0}, \theta\right] \times \mathbb{R}^{n} \times \mathbb{R}^{m} \rightarrow \mathbb{R}^{n}$ satisfy the conditions $2 . \mathrm{A}$ and 2 . B. Then

$$
\begin{aligned}
\|a(t, x)\| & \leq L_{0}\|x\|+\gamma_{0}, \\
\|K(t, s, x, u)\| & \leq\left[L_{2}+\left(H_{2}+H_{3}\right)\|u\|\right]\|x\|+L_{3}\|u\|+\gamma_{1}
\end{aligned}
$$

for every $(t, s, x, u) \in\left[t_{0}, \theta\right] \times\left[t_{0}, \theta\right] \times \mathbb{R}^{n} \times \mathbb{R}^{m}$ where $L_{0}, L_{2}, L_{3}, H_{2}$ and $H_{3}$ are defined in condition 2.B, $\gamma_{0}$ and $\gamma_{1}$ are defined by (4.1) and (4.2) respectively.

Proof. Let $(t, s, x, u) \in\left[t_{0}, \theta\right] \times\left[t_{0}, \theta\right] \times \mathbb{R}^{n} \times \mathbb{R}^{m}$ be an arbitrarily chosen. Condition 2.B implies that

$$
\|K(t, s, x, u)-K(t, s, 0,0)\| \leq\left(L_{2}+H_{2}\|u\|\right)\|x\|+\left(L_{3}+H_{3}\|x\|\right)\|u\|
$$

and hence

$$
\begin{aligned}
\|K(t, s, x, u)\| & \leq\left(L_{2}+H_{2}\|u\|\right)\|x\|+\left(L_{3}+H_{3}\|x\|\right)\|u\|+\|K(t, s, 0,0)\| \\
& \leq\left[L_{2}+\left(H_{2}+H_{3}\right)\|u\|\right]\|x\|+L_{3}\|u\|+\gamma_{1},
\end{aligned}
$$

where $\gamma_{1}$ is defined by (4.2).

The validity of inequality $\|a(t, x)\| \leq L_{0}\|x\|+\gamma_{0},(t, x) \in\left[t_{0}, \theta\right] \times \mathbb{R}^{n}$, is proved analogously.

Denote

$$
\begin{aligned}
& \rho_{*}=\left(\gamma_{0}+\gamma_{1}\left(\theta-t_{0}\right) \lambda+\lambda L_{3}\left(\theta-t_{0}\right)^{\frac{p-1}{p}} \mu_{0}\right) /\left(1-L_{0}\right), \\
& P(\lambda)=\lambda\left(L_{2}\left(\theta-t_{0}\right)+\left(H_{2}+H_{3}\right)\left(\theta-t_{0}\right)^{\frac{p-1}{p}} \mu_{0}\right), \\
& r_{*}=\rho_{*} \exp \left[P(\lambda) /\left(1-L_{0}\right)\right] .
\end{aligned}
$$

The following theorem specifies boundedness of the set of trajectories. 
Theorem 2. For every $x(\cdot) \in \mathbf{X}_{p}$ the inequality $\|x(\cdot)\|_{C} \leq r_{*}$ holds.

Proof. Let us choose an arbitrary $x(\cdot) \in \mathbf{X}_{p}$. Then there exists $u(\cdot) \in U_{p}$ such that

$$
x(t)=a(t, x(t))+\lambda \int_{t_{0}}^{t} K(t, s, x(s), u(s)) d s, \quad t \in\left[t_{0}, \theta\right] .
$$

Proposition 2 implies that

$$
\begin{aligned}
\|x(t)\| \leq & L_{0}\|x(t)\|+\gamma_{0} \\
& +\lambda \int_{t_{0}}^{t}\left(\left[L_{2}+\left(H_{2}+H_{3}\right)\|u(s)\|\right]\|x(s)\|+L_{3}\|u(s)\|+\gamma_{1}\right) d s \\
\leq & L_{0}\|x(t)\|+\gamma_{0}+\gamma_{1}\left(\theta-t_{0}\right) \lambda+\lambda L_{3}\left(\theta-t_{0}\right)^{\frac{p-1}{p}} \mu_{0} \\
& +\lambda \int_{t_{0}}^{t}\left[L_{2}+\left(H_{2}+H_{3}\right)\|u(s)\|\right]\|x(s)\| d s
\end{aligned}
$$

for every $t \in\left[t_{0}, \theta\right]$. Since $L_{0} \in[0,1)$ then the last inequality and (4.3) yields

$$
\begin{aligned}
\|x(t)\| \leq & \left(\gamma_{0}+\gamma_{1}\left(\theta-t_{0}\right) \lambda+\lambda L_{3}\left(\theta-t_{0}\right)^{\frac{p-1}{p}} \mu_{0}\right) /\left(1-L_{0}\right) \\
& +\frac{\lambda}{1-L_{0}} \int_{t_{0}}^{t}\left[L_{2}+\left(H_{2}+H_{3}\right)\|u(s)\|\right]\|x(s)\| d s \\
= & \rho_{*}+\frac{\lambda}{1-L_{0}} \int_{t_{0}}^{t}\left[L_{2}+\left(H_{2}+H_{3}\right)\|u(s)\|\right]\|x(s)\| d s
\end{aligned}
$$

for every $t \in\left[t_{0}, \theta\right]$. From (4.4), (4.5), (4.6) and Gronwall's inequality we obtain

$$
\begin{aligned}
\|x(t)\| & \leq \rho_{*} \exp \left[\frac{\lambda}{1-L_{0}} \int_{t_{0}}^{t}\left(L_{2}+\left(H_{2}+H_{3}\right)\|u(s)\|\right) d s\right] \\
& =\rho_{*} \exp \left[\frac{\lambda}{1-L_{0}}\left(L_{2}\left(t-t_{0}\right)+\left(H_{2}+H_{3}\right) \int_{t_{0}}^{t}\|u(s)\| d s\right)\right] \\
& \leq \rho_{*} \exp \left[\frac{\lambda}{1-L_{0}}\left(L_{2}\left(\theta-t_{0}\right)+\left(H_{2}+H_{3}\right)\left(\theta-t_{0}\right)^{\frac{p-1}{p}} \mu_{0}\right)\right] \\
& =\rho_{*} \exp \left[P(\lambda) /\left(1-L_{0}\right)\right]=r_{*}
\end{aligned}
$$

for every $t \in\left[t_{0}, \theta\right]$ which completes the proof.

Thus, from Theorem 2 we have that the set of trajectories $\mathbf{X}_{p}$ of the system $(2.1)$ is a bounded subset of the space $C\left(\left[t_{0}, \theta\right] ; \mathbb{R}^{n}\right)$. In particular, from Theorem 2 it follows the validity of the following corollary.

Corollary 1. The inclusion $\mathbf{X}_{p}(t) \subset B_{n}\left(r_{*}\right)$ holds for every $t \in\left[t_{0}, \theta\right]$ where the set $\mathbf{X}_{p}(t)$ is defined by (2.3), the number $r_{*}>0$ is defined by $(4.5), B_{n}\left(r_{*}\right)=$ $\left\{x \in \mathbb{R}^{n}:\|x\| \leq r_{*}\right\}$. 


\section{Precompactness of the Set of Trajectories}

In this section precompactness of the set of trajectories is studied.

Denote $D_{1}=\left[t_{0}, \theta\right] \times B_{n}\left(r_{*}\right)$,

$$
\begin{gathered}
\omega_{0}(\Delta)=\max \left\{\left\|a\left(t_{2}, x\right)-a\left(t_{1}, x\right)\right\|:\left|t_{2}-t_{1}\right| \leq \Delta,\right. \\
\left.\left(t_{1}, x\right) \in D_{1}, \quad\left(t_{2}, x\right) \in D_{1}\right\}, \\
\varphi(\Delta)=\frac{1}{1-L_{0}}\left\{\omega_{0}(\Delta)+\lambda\left[L_{1}\left(\theta-t_{0}\right)+2 H_{1}\left(\theta-t_{0}\right)^{\frac{p-1}{p}} \mu_{0}\right] \Delta\right. \\
\left.+\lambda\left(L_{2} r_{*}+\gamma_{1}\right) \Delta+\lambda \mu_{0}\left[\left(H_{2}+H_{3}\right) r_{*}+L_{3}\right] \Delta^{\frac{p-1}{p}}\right\},
\end{gathered}
$$

where $r_{*}$ is defined by (4.5). By virtue of condition 2.A the function $a(\cdot)$ is continuous. Then $\omega_{0}(\Delta) \rightarrow 0, \varphi(\Delta) \rightarrow 0$ as $\Delta \rightarrow 0^{+}$.

The Hausdorff distance between the sets $U \subset \mathbb{R}^{n}$ and $V \subset \mathbb{R}^{n}$ is denoted by $h(U, V)$ and defined as

$$
h(U, V)=\max \left\{\sup _{u \in U} d(u, V), \sup _{v \in V} d(v, U)\right\},
$$

where $d(u, V)=\inf \{\|u-v\|: v \in V\}$.

Proposition 3. For every $x(\cdot) \in \mathbf{X}_{p}, t_{1} \in\left[t_{0}, \theta\right], t_{2} \in\left[t_{0}, \theta\right]$ the inequality

$$
\left\|x\left(t_{2}\right)-x\left(t_{1}\right)\right\| \leq \varphi\left(\left|t_{2}-t_{1}\right|\right)
$$

holds and hence $h\left(\mathbf{X}_{p}\left(t_{2}\right), \mathbf{X}_{p}\left(t_{1}\right)\right) \leq \varphi\left(\left|t_{2}-t_{1}\right|\right)$, where $\mathbf{X}_{p}\left(t_{1}\right)$ and $\mathbf{X}_{p}\left(t_{2}\right)$ are defined by (2.3).

Proof. Let $x(\cdot) \in \mathbf{X}_{p}$ be an arbitrarily chosen trajectory of the system (2.1). Then there exists $u(\cdot) \in U_{p}$ such that

$$
x(t)=a(t, x(t))+\lambda \int_{t_{0}}^{t} K(t, s, x(s), u(s)) d s, \quad t \in\left[t_{0}, \theta\right] .
$$

Now let $t_{1} \in\left[t_{0}, \theta\right], t_{2} \in\left[t_{0}, \theta\right]$ and let $t_{2}>t_{1}$. Then

$$
\begin{aligned}
\left\|x\left(t_{2}\right)-x\left(t_{1}\right)\right\| \leq & \left\|a\left(t_{2}, x\left(t_{2}\right)\right)-a\left(t_{1}, x\left(t_{2}\right)\right)\right\|+\left\|a\left(t_{1}, x\left(t_{2}\right)\right)-a\left(t_{1}, x\left(t_{1}\right)\right)\right\| \\
& +\lambda \int_{t_{0}}^{t_{1}}\left\|K\left(t_{2}, s, x(s), u(s)\right)-K\left(t_{1}, s, x(s), u(s)\right)\right\| d s \\
& +\lambda \int_{t_{1}}^{t_{2}}\left\|K\left(t_{2}, s, x(s), u(s)\right)\right\| d s .
\end{aligned}
$$

Since $u(\cdot) \in U_{p}$, then by virtue of condition 2.B we get

$$
\begin{aligned}
& \int_{t_{0}}^{t_{1}}\left\|K\left(t_{2}, s, x(s), u(s)\right)-K\left(t_{1}, s, x(s), u(s)\right)\right\| d s \\
& \quad \leq \int_{t_{0}}^{t_{1}}\left(L_{1}+2 H_{1}\|u(s)\|\right)\left(t_{2}-t_{1}\right) d s \\
& \quad \leq\left[L_{1}\left(\theta-t_{0}\right)+2 H_{1}\left(\theta-t_{0}\right)^{\frac{p-1}{p}} \mu_{0}\right]\left(t_{2}-t_{1}\right) .
\end{aligned}
$$


Taking into consideration that $u(\cdot) \in U_{p}$, we obtain from Proposition 2 and Theorem 2 that

$$
\begin{aligned}
& \int_{t_{1}}^{t_{2}}\left\|K\left(t_{2}, s, x(s), u(s)\right)\right\| d s \\
& \quad \leq \int_{t_{1}}^{t_{2}}\left[\left(L_{2}+\left(H_{2}+H_{3}\right)\|u(s)\|\right)\|x(s)\|+L_{3}\|u(s)\|+\gamma_{1}\right] d s \\
& \quad \leq \int_{t_{1}}^{t_{2}}\left[\left(L_{2}+\left(H_{2}+H_{3}\right)\|u(s)\|\right) r_{*}+L_{3}\|u(s)\|+\gamma_{1}\right] d s \\
& \quad=\left(L_{2} r_{*}+\gamma_{1}\right)\left(t_{2}-t_{1}\right)+\int_{t_{1}}^{t_{2}}\left[\left(H_{2}+H_{3}\right) r_{*}+L_{3}\right]\|u(s)\| d s \\
& \quad=\left(L_{2} r_{*}+\gamma_{1}\right)\left(t_{2}-t_{1}\right)+\left[\left(H_{2}+H_{3}\right) r_{*}+L_{3}\right] \int_{t_{1}}^{t_{2}}\|u(s)\| d s \\
& \quad \leq\left(L_{2} r_{*}+\gamma_{1}\right)\left(t_{2}-t_{1}\right)+\mu_{0}\left[\left(H_{2}+H_{3}\right) r_{*}+L_{3}\right]\left(t_{2}-t_{1}\right)^{\frac{p-1}{p}} .
\end{aligned}
$$

Condition 2.B implies

$$
\left\|a\left(t_{1}, x\left(t_{2}\right)\right)-a\left(t_{1}, x\left(t_{1}\right)\right)\right\| \leq L_{0}\left\|x\left(t_{2}\right)-x\left(t_{1}\right)\right\|,
$$

where $L_{0} \in[0,1)$.

Since $(t, x(t)) \in D_{1}$ for every $t \in\left[t_{0}, \theta\right]$, we get from (5.1) that

$$
\left\|a\left(t_{2}, x\left(t_{2}\right)\right)-a\left(t_{1}, x\left(t_{2}\right)\right)\right\| \leq \omega_{0}\left(\left|t_{2}-t_{1}\right|\right) .
$$

Now from (5.3)-(5.7) we conclude

$$
\begin{aligned}
& \left\|x\left(t_{2}\right)-x\left(t_{1}\right)\right\| \leq L_{0}\left\|x\left(t_{2}\right)-x\left(t_{1}\right)\right\|+\omega_{0}\left(\left|t_{2}-t_{1}\right|\right) \\
& \quad+\lambda\left[L_{1}\left(\theta-t_{0}\right)+2 H_{1}\left(\theta-t_{0}\right)^{\frac{p-1}{p}} \mu_{0}\right]\left(t_{2}-t_{1}\right) \\
& \quad+\lambda\left(L_{2} r_{*}+\gamma_{1}\right)\left(t_{2}-t_{1}\right)+\lambda \mu_{0}\left[\left(H_{2}+H_{3}\right) r_{*}+L_{3}\right]\left(t_{2}-t_{1}\right)^{\frac{p-1}{p}} .
\end{aligned}
$$

Since $L_{0} \in[0,1)$, then $(5.2)$ and (5.8) yield

$$
\begin{aligned}
\| x\left(t_{2}\right) & -x\left(t_{1}\right) \| \leq \frac{1}{1-L_{0}}\left\{\omega_{0}\left(\left|t_{2}-t_{1}\right|\right)+\lambda\left[L_{1}\left(\theta-t_{0}\right)+2 H_{1}\left(\theta-t_{0}\right)^{\frac{p-1}{p}} \mu_{0}\right]\right. \\
& \left.\times\left(t_{2}-t_{1}\right)+\lambda\left(L_{2} r_{*}+\gamma_{1}\right)\left(t_{2}-t_{1}\right)+\lambda \mu_{0}\left[\left(H_{2}+H_{3}\right) r_{*}+L_{3}\right]\left(t_{2}-t_{1}\right)^{\frac{p-1}{p}}\right\} \\
& =\varphi\left(\left|t_{2}-t_{1}\right|\right) .
\end{aligned}
$$

Since $\varphi(\Delta) \rightarrow 0$ as $\Delta \rightarrow 0^{+}$then we obtain from Proposition 3 the validity of the following corollary.

Corollary 2. Set valued map $t \rightarrow \mathbf{X}_{p}(t), t \in\left[t_{0}, \theta\right]$, is continuous.

Proposition 3 also implies the validity of the following theorem.

Theorem 3. The set of trajectories $\mathbf{X}_{p}$ is a precompact subset of the space $C\left(\left[t_{0}, \theta\right], \mathbb{R}^{n}\right)$. 
Proof. According to the Theorem 2 the set of trajectories of the system (2.1) with constraint (2.2), i.e. the set $\mathbf{X}_{p}$ is a bounded subset of the space $C\left(\left[t_{0}, \theta\right], \mathbb{R}^{n}\right)$.

Let us choose an arbitrary $\varepsilon>0$. Since $\varphi(\Delta) \rightarrow 0$ as $\Delta \rightarrow 0^{+}$, where the function $\varphi(\cdot):[0, \infty) \rightarrow[0, \infty)$ is defined by $(5.2)$, then for $\varepsilon>0$ there exists $\delta(\varepsilon)>0$ such that $\varphi(\Delta)<\varepsilon$ for every $\Delta<\delta(\varepsilon)$.

Now let $x(\cdot) \in \mathbf{X}_{p}$ be an arbitrarily chosen trajectory and $t_{1} \in\left[t_{0}, \theta\right]$, $t_{2} \in\left[t_{0}, \theta\right]$ be such that $\left|t_{2}-t_{1}\right|<\delta(\varepsilon)$. Then $\varphi\left(\left|t_{2}-t_{1}\right|\right)<\varepsilon$ and we obtain from Proposition 3 that

$$
\left\|x\left(t_{2}\right)-x\left(t_{1}\right)\right\| \leq \varphi\left(\left|t_{2}-t_{1}\right|\right)<\varepsilon .
$$

Thus the set of trajectories $\mathbf{X}_{p}$ is the set of equicontinuous functions. From Arzela-Ascoli theorem we have the proof of the theorem.

Note that the set of trajectories $\mathbf{X}_{p}$ is not a closed subset of the space $C\left(\left[t_{0}, \theta\right], \mathbb{R}^{n}\right)$. To illustrate this assertion we use examples, given in [2] and [5].

Example 1. Consider the controllable system whose behaviour is described by the integral equations

$$
\left\{\begin{array}{l}
x(t)=\int_{0}^{t}\left[-y^{2}(s)+u^{2}(s)\right] d s, \\
y(t)=\int_{0}^{t} u(s) d s
\end{array}\right.
$$

where $(x, y) \in \mathbb{R}^{2}$ is the state vector, $u \in \mathbb{R}$ is a control vector, $t \in[0,1]$. Every $u(\cdot) \in L_{2}([0,1], \mathbb{R})$ which satisfies the inequality $\int_{0}^{1} u^{2}(t) d t \leq 1$ is called an admissible control function. The set of all admissible control functions is denoted by $U_{2}^{*}$. Then

$$
U_{2}^{*}=\left\{u(\cdot) \in L_{2}([0,1] ; \mathbb{R}):\|u(\cdot)\|_{2} \leq 1\right\} .
$$

The set of trajectories of the system (5.9) generated by all admissible control functions $u(\cdot) \in U_{2}^{*}$ is denoted by $\mathbf{X}_{2}^{*}$.

The set of trajectories $\mathbf{X}_{2}^{*}$ is bounded (see [5]). Let the trajectory of the system $(5.9)\left(x_{k}(\cdot), y_{k}(\cdot)\right) \in \mathbf{X}_{2}^{*}$ be generated by the admissible control functions $u_{k}(\cdot) \in U_{2}^{*}$ where

$$
u_{k}(t)= \begin{cases}1, & t \in\left[\frac{2 i}{2 k}, \frac{2 i+1}{2 k}\right), \\ -1, & t \in\left[\frac{2 i+1}{2 k}, \frac{2 i+2}{2 k}\right), i=0,1, \ldots, k-1\end{cases}
$$

and $k=1,2, \ldots$

It is possible to verify that $\left(x_{k}(\cdot), y_{k}(\cdot)\right) \rightarrow\left(x_{*}(\cdot), y_{*}(\cdot)\right)$ as $k \rightarrow \infty$ where $x_{*}(t)=t, y_{*}(t)=0$ for every $t \in[0,1]$, but $\left(x_{*}(\cdot), y_{*}(\cdot)\right) \notin \mathbf{X}_{2}^{*}$. Moreover, $\left(x_{k}(1), y_{k}(1)\right) \rightarrow(1,0)$ as $k \rightarrow \infty$, but $(1,0) \notin \mathbf{X}_{2}^{*}(1)=\left\{(x(1), y(1)) \in \mathbb{R}^{2}:\right.$ $\left.(x(\cdot), y(\cdot)) \in \mathbf{X}_{2}^{*}\right\}($ see $[2,5])$.

So, the set of trajectories of the system (5.9), i.e. the set $\mathbf{X}_{2}^{*}$ is not a closed subset of the space $C\left([0,1], \mathbb{R}^{2}\right)$. The section of the set of trajectories, i.e. the set $\mathbf{X}_{2}^{*}(1)$ also is not a closed subset of the space $\mathbb{R}^{2}$. 


\section{Conclusion}

The existence of the optimal trajectories is one of the important problems of the control systems theory. Under appropriate conditions, precompactness of the set of trajectories allows to forecast the existence of the $\varepsilon$-optimal trajectories of the control system. If the behaviour of the control system is described by a nonlinear Volterra integral equation and it has continuous payoff functional and limited control resources, which are exhausted by consuming, such as fuel or money, then precompactness theorem guarantees the existence of the $\varepsilon$-optimal trajectories.

\section{References}

[1] T.A. Burton. Six integral equations and a flexible Lyapunov functional. Proc. Inst. Math. Mech. Ural Branch of Russian Acad. Sci., 16(5):241-252, 2010.

[2] A.F. Filippov. Differential Equations with Discontinuous Right-Hand Sides. Kluwer, Dordrecht, 1988.

[3] I.G. Gohberg and M.G. Krein. Theory and Applications of Volterra Operators in Hilbert Space. Amer. Math. Soc., Providence, RI, 1970.

[4] Kh.G. Guseinov. Approximation of the attainable sets of the nonlinear control systems with integral constraint on controls. Nonlinear Anal., 71:622-645, 2009. http://dx.doi.org/10.1016/j.na.2008.10.097.

[5] Kh.G. Guseinov and A.S. Nazlipinar. On the continuity properties of the attainable sets of nonlinear control systems with integral constraint on controls. Abstr. Appl. Anal., 2008. http://dx.doi.org/10.1155/2008/295817. Article ID 295817, $14 \mathrm{pp}$.

[6] M.A. Krasnoselskii and S.G. Krein. On the principle of avaraging in nonlinear mechanics. Uspekhi Mat. Nauk, 10(3):147-153, 1955. (In Russian)

[7] N.N. Krasovskii. Theory of Control of Motion: Linear Systems. Nauka, Moscow, 1968. (In Russian)

[8] R.K. Miller. Nonlinear Volterra Integral Equations. Benjamin, Inc., Menlo Park, CA, 1971.

[9] A.D. Polyanin and A.V. Manzhurov. Handbook of Integral Equation. CRC Press, Boca Raton, FL, 1998.

[10] A.I. Subbotin and V.N. Ushakov. Alternative for an encounter-evasion differential game with integral constraints on the players controls. J. Appl. Math. Mech., 39(3):367-375, 1975. http://dx.doi.org/10.1016/0021-8928(75)90001-5.

[11] V.I. Ukhobotov. One Dimensional Projection Method in Linear Differential Games with Integral Constraints. Chelyabinsk State University Press, Chelyabinsk, 2005. (In Russian)

[12] M. Väth. Volterra and Integral Equations of Vector Functions. M. Deccer. Inc., New York, 2000. 